\title{
Risk Factors for Ventricular Tachyarrhythmic Events in Patients Without Left Bundle Branch Block Who Receive Cardiac Resynchronization Therapy
}

\author{
Arwa Younis ${ }^{1}$, Mehmet Aktas ${ }^{1}$, Wojciech Zareba ${ }^{2}$, Scott McNitt ${ }^{2}$, Valentina Kutyifa ${ }^{1}$, and \\ Ilan Goldenberg ${ }^{2}$ \\ ${ }^{1}$ University of Rochester Medical Center \\ ${ }^{2}$ University of Rochester
}

September 21, 2020

\begin{abstract}
Introduction: Cardiac resynchronization therapy (CRT) may be proarrhtyhmic in patients with non-left bundle branch block (non-LBBB). We hypothesized that combined assessment of risk factors (RF) for ventricular tachyarrhythmias (VTA) can be used to stratify non-LBBB patients for CRT implantation. Methods: The study comprised 412 non-LBBB patients from MADIT-CRT randomized to CRT-D $(\mathrm{n}=215)$ vs. ICD-only $(\mathrm{n}=197)$. Best-subset regression analysis was performed to identify RF associated with increased VTA-risk in CRT-D patients without LBBB. The primary endpoint was first occurrence of sustained VTA during follow-up. Secondary endpoints included VTA/death, and appropriate shock. Results: Four RFs were associated with increased VTA risk: Blood Urea Nitrogen $>25 \mathrm{mg} / \mathrm{dl}$, ejection-fraction $<20 \%$, prior non-sustained VT, and female gender. Among CRT-D patients, 114 (53\%) had no RF, while 101 (47\%) had [?] 1 RF. The 4-year cumulative probability of VTA was higher among those with [?] 1 RF compared with those without RF ( $40 \%$ vs. $14 \%, \mathrm{p}<0.001$ ). Multivariate analysis showed that in patients without RF, treatment with CRT-D was associated with a $61 \%$ reduction in VTA compared with ICDonly therapy $(\mathrm{p}=0.002)$, whereas among patients with [?] $1 \mathrm{RF}$ treatment with CRT-D was associated with a corresponding $73 \%(\mathrm{p}=0.025)$ risk-increase. Consistent results were observed when the secondary endpoints of VTA/death and appropriate ICD shocks were assessed. Conclusion: Combined assessment of factors associated with increased risk for VTA can be used for improved selection of non-LBBB patients for CRT-D.
\end{abstract}

\section{Introduction}

Cardiac resynchronization therapy (CRT) was shown to improve symptoms and decrease mortality in patients with heart failure (HF) with a wide QRS ${ }^{1-3}$. However, data on the benefit of the device by QRS morphology are conflicting. Current ESC Guidelines provide a Class II recommendation for CRT-D implantation in patients without left bundle branch block (LBBB) ${ }^{4}$, yet several randomized studies have reported on the relative absence of clinical benefit of CRT in non-LBBB patients compared to those with LBBB. ${ }^{5}$ Therefore, studies on improved risk stratification for CRT-D in patients without LBBB are needed.

Recent studies have attempted to identify subgroups of non-LBBB patients who may respond clinically to CRT, such as those with prolonged PR intervals ([?]230 ms) ${ }^{6}$ or those with (RBBB) with concomitant left-sided delay and those with significant burden of right ventricular pacing, with conflicting results ${ }^{7}$. Our data from MADIT-CRT suggest a possible harm, with increased risk for ventricular tachyarrhythmias (VTA) among non-LBBB patients implanted with a CRT-D device compared with implantable cardioverterdefibrillator (ICD)-only therapy. ${ }^{8,9}$ The exact pathophysiology behind this observation remains unclear, however these findings indicate a possible pro-arrhythmic effect of CRT in this population that may lead to subsequent adverse outcomes. ${ }^{10-13}$ 
Accordingly, the primary aim of the present study was to identify risk factors (RF) for VTA in patients without LBBB who were enrolled in MADIT-CRT. We hypothesized that combined RF assessment can be used to identify those in whom CRT implantation would beneficial vs. proarrhythmic.

\section{Methods}

\section{Study Population}

The present study cohort comprised 412 patients from MADIT-CRT with non-LBBB and complete information on baseline clinical, echocardiographic, and laboratory variables was available. The rationale and design of the MADIT-CRT trial was previously published. ${ }^{14}$ Briefly, MADIT-CRT was designed to determine whether CRT with a defibrillator (CRT-D) would reduce the risk of death or HF events in patients with mild HF symptoms, a reduced ejection fraction, and wide QRS complex compared with ICD therapy. Patients were randomly assigned in a 3:2 ratio to receive either CRT-D or ICD. From December 22, 2004, through April 23, 2008, a total of 1820 patients were enrolled at 110 hospital centers. The protocol was approved by the institutional review board at each of the participating centers. Patients of either sex who were at least 21 years of age were enrolled in the study if they had ischemic cardiomyopathy (NYHA class I or II) or non-ischemic cardiomyopathy (NYHA class II only), sinus rhythm, an ejection fraction of [?]0.30, and prolonged intraventricular conduction with a QRS duration of [?]130 milliseconds. All eligible subjects met guideline indications for ICD therapy. Of the 412 patients without LBBB who were included in the present study, 215 were randomized to CRT-D therapy and 197 were randomized to ICD-only therapy.

\section{End points and rhythm adjudication}

The primary end point of this study was the first occurrence of VTA. Secondary endpoints were; composite of VTA or death (whichever comes first) and appropriate defibrillator shock therapy. All device therapies delivered were blindly adjudicated by 2 experienced electrophysiologists. VTA were defined as any ICD-recorded, treated or monitored sustained ventricular tachycardia (VT) faster or equal to $180 \mathrm{bpm}$ or ventricular fibrillation (VF).

\section{Statistical analysis}

Continuous variables are expressed as mean \pm SD. Categorical data are summarized as frequencies and percentages. Baseline clinical characteristics were compared between patients with ICD, low risk CRT-D and high risk CRT-D, using Wilcoxon ranked sum test for continuous variables and $\mathrm{Chi}^{2}$ - test for dichotomous variables, as appropriate.

Step 1- covariate selection: We included 29 potential clinical, electrocardiographic, and laboratory binary risk factors for VTA (Listed in eTable A in the Online Appendix). Numeric variables were made binary by the use of cut points with the goal of finding a simple, easily implemented predictors to be derived from them. Thresholds for categorization of numeric variables were based on the mean or clinical relevance. Univariate relationships between candidate covariates and a further event were assessed by t tests ( 2 for binary responses). The covariates with values of $\mathrm{P}<0.20$ were further evaluated by carrying out a best-subset regression analysis, examining the models created from all possible combinations of predictor variables, and using a penalty of 3.84 on the likelihood ratio 2 value for any additional factor included (corresponds to a $\mathrm{P}$ of $5 \%$ for a 1 -df 2 test).

Step 2 - Creating the groups: For the main analysis, CRT-D patients were grouped in two groups based on the presence or absence of RF. In a secondary analysis, we further grouped CRT-D patients into 3 groups; (i) No RF, (ii) One RF, and (iii) two or more RF. Since the identified CRT-D RF were not associated with outcomes in the ICD group (online supplemental Figure A), ICD patients were not further grouped and were included as a control group

Step 3 - Outcomes by score analysis: Kaplan-Meier estimates for any VTA, VTA or death, and appropriate shock in patients with ICD and CRT-D, stratified by their risk, were determined and statistically evaluated 
with the log-rank test. Multivariate Cox proportional hazards regression analyses were carried out in the subgroups for the assessment of the primary and secondary end points. The following covariates were included in the Cox regression models: age, gender, QRS width, New York Heart Association class NYHA, creatinine, left ventricular ejection fraction, diabetes, and ischemic origin.

All statistical tests were two-sided, a p-value of $<0.05$ was considered statistically significant. Analyses were carried out with SAS software (version 9.4, SAS institute, Cary, North Carolina).

\section{Results}

Predictors of increased risk for ventricular arrhythmia and risk score

A best-subset regression analysis in the CRT-D arm of the trial identified 4 factors (from the 29 candidate covariates listed in Table A in the online-only data supplement) as being associated with increased risk for VTA (eTable B). These factors were blood urea nitrogen (BUN) $>25 \mathrm{mg} / \mathrm{dl}$, left ventricular ejection fraction $<20 \%$, prior non sustained ventricular tachycardia (NSVT), and female gender.

The baseline clinical characteristics of study patients by the presence of VTA RF in CRT-D patients and the total ICD group are presented in eTable C. Mean age of the study patients was $65 \pm 10$ years, and $10 \%$ were female. Patients with [?] 1 RF for VTA comprised $47 \%$ of the CRT-D population, and had similar baseline characteristics as those without RF, with the exception of a higher baseline creatinine, and a higher frequency of diuretic use. Among the group of CRT-D patients with [?] 1RF, $82 \%$ had only 1 RF, while 17 patients (17\%) had two RF, and only two patients (1\%) had three RF.

\section{Association of VTA RFs with outcomes in CRT-D patients without LBBB}

Figure 1A shows that among non-LBBB patients with CRT-D, the 4-year cumulative probability of VTA was significantly higher among those with [?]1 RF when compared to those without RF (40\% vs. $14 \%, \mathrm{p}<0.001$, respectively). Similarly, patients with [?]1 RF experienced a significant higher rate of the secondary outcomes measures of VTA or death $(51 \%$ vs. $22 \%$, respectively log-rank $\mathrm{p}<0.001$ for the overall difference during follow-up [Figure 1B]) and appropriate ICD shock (28\% vs. $7 \%$, respectively; $\mathrm{p}=0.002$ [Figure 1C]).

The results of the CRT-D-only multivariate Cox regression models are presented in Table 1. Among nonLBBB patients with CRT-D, the presence of one or more RF was associated with $>3.5$-fold $(\mathrm{p}<0.001)$ increased risk for VTA compared with no RF. Furthermore, patients who had 2 or more RF experienced a $>9$-fold $(\mathrm{p}<0.001)$ increased risk for VTA compared with those with no RF. Similar results were shown for the secondary endpoints of VTA/death and appropriate ICD shock (Table 1).

\section{Benefit of CRT-D vs. ICD-only therapy by VTA risk-score}

Among all non-LBBB patients enrolled in MADIT-CRT (i.e. with ICD or CRT-D), the 4-year cumulative probability of VTA was highest among CRT-D patients with RF (40\%); intermediate in the ICD group (33\%); and lowest among CRT-D without RF ([14\%], log-rank $\mathrm{p}<0.001$ for the overall difference during follow-up [Figure 2; left panel]). Similarly, the 4-year cumulative probability of the composite of death or VTA was $51 \%$ in CRT-D patients with RF, $42 \%$ in the ICD-only group, and $22 \%$ in the CRT-D group without RF (log-rank $\mathrm{p}<0.001$ for the overall difference during follow-up [Figure 2: middle panel]); and the respective rates of appropriate defibrillator shock therapy were $28 \%, 19 \%$, and $7 \%$ respectively (log-rank p $=0.008$ for the overall difference during follow-up [Figure 2: right panel]).

Multivariate analysis showed that among non-LBBB patients with no RF, treatment with CRT-D therapy was associated with a statistically significant $61 \%(\mathrm{p}=0.002)$ reduction in the risk of the primary VTA endpoint compared with ICD-only therapy. Similar results were shown for the secondary endpoints, wherein treatment with CRT was associated with a significant $50 \%(\mathrm{p}=0.011)$ reduction in VTA/death, and a significant $52 \%(\mathrm{p}=0.079)$ reduction in the risk of appropriate ICD shock therapy (Table 2). In contrast, among non-LBBB patients who had one or more RF, treatment with CRT-D was associated with a statistically significant $73 \%(\mathrm{p}=0.025)$ increase in the risk of VTA; a $72 \%(\mathrm{p}=0.014)$ increase in the composite endpoint 
of VTA/death; and a 91\% ( $\mathrm{p}=0.043)$ increase in the risk of appropriate defibrillator shock therapy (Table 2).

\section{Discussion}

In the present study we provide several important insights on predictors for VTA outcomes in CRT-D patients with non-LBBB, in whom data on the potential benefit of the device are limited and conflicting. First, we identified 4 simple baseline clinical factors $(\mathrm{BUN}>25 \mathrm{mg} / \mathrm{dl}, \mathrm{EF}<20 \%$, prior NSVT, and female gender) that were associated with increased risk for VTA in CRT-D patients with non-LBBB. Second, we have shown that among patients without any of the identified RF, treatment with CRT-D was associated with a significant reduction in the risk of arrhythmic events compared with ICD-only therapy, including any VTA, VTA/death, and appropriate defibrillator shocks. In contrast, among patients with at least one RF, treatment with CRT-D was associated with a possible proarrhytmic effect, wherein the risk of any VTA, VTA/death, and appropriate defibrillator shocks, was significantly higher among patients implanted with a CRT-D device compared with those treated only with an ICD. These findings suggest that combined assessment of simple baseline clinical parameters can be used to distinguish CRT-D candidates without LBBB who may benefit from biventricular pacing compared with those in whom CRT-D may be possibly be associated with a harmful proarrhythmic effect.

In a meta-analysis of the five landmark randomized controlled trials evaluating CRT in the HF population, with a total of 1766 patients with non-LBBB QRS morphology at baseline, the use of CRT was not associated with a significant reduction in mortality or HF hospitalizations. ${ }^{15}$ Moreover, among non-LBBB patients the use of CRT-D was suggested to be harmful and possibly associated with increased all-cause mortality when compared with ICD alone. ${ }^{8,16}$ The mechanism related to the observed trend in mortality increase associated with CRT-D in non-LBBB patients remains unclear. We have previously shown that in MADIT-CRT non-LBBB patients who were randomized to CRT-D therapy experienced a significant 3.6fold $(\mathrm{p}=0.009)$ increased risk for recurrent VTAs compared to ICD-only patients. ${ }^{17}$ These findings may be due to a pro-arrhythmic effect of CRT among patients without LBBB leading to increased risk associated with the device in this population. These data are consistent with previous studies, including a large comprehensive meta-analysis, which reported on the pro-arrhythmic effect of CRT among non-responders to the device. ${ }^{10-13} \mathrm{~A}$ potential mechanism for the pro-arrhythmic role of CRT-D in non-LBBB patients is the reversal of left ventricular activation and increased transmural dispersion of repolarization with epicardial pacing, thus allowing for the development of early after depolarizations and re-entrant circuits. ${ }^{12}$ Despite the above-mentioned observations, current ESC Guidelines provide a Class II recommendation (with a or b level based on QRS duration) for CRT in non-LBBB patients. ${ }^{4}$ Based on this recommendation, approximately one quarter of CRT-D implants from 2012 to 2015 through the National Cardiovascular Data Registry for Implantable Cardioverter-Defibrillators were in patients who had a non-LBBB morphology at baseline. ${ }^{18}$

\section{Predictors for ventricular arrhythmia}

In the present study we identified 4 factors that were associated with increased risk of VTA, including $\mathrm{BUN}>25 \mathrm{mg} / \mathrm{dl}, \mathrm{EF}<20 \%$, prior NSVT, and female gender. Reduced EF and prior NSVT reflect substrate and vulnerability to sustained VTA in patients with CRT-D,${ }^{19,20}$ while elevated BUN is correlated with diuretic use, and may therefore be a marker of more advanced HF rather than an indicator of renal dysfunction, as creatinine and eGFR did not enter the final prediction model.

Several previous publications have suggested that women may respond better to CRT with subsequent lower mortality than men. ${ }^{21,22}$ Our study suggests that among patients without LBBB, the effect of sex may be different, and that non-LBBB women may experience increased VTA risk with CRT-D. These data are consistent with our prior findings from the long-term follow-up of MADIT-CRT, in which we have shown that the pronounced benefit of CRT for the endpoint of all-cause mortality among women was restricted to those with LBBB, whereas CRT-D was associated with a trend to a mortality risk increase among women without LBBB.

\section{The effect of CRT in non-LBBB}


In contemporary practice, the use of CRT in non-LBBB patients remains controversial, mainly due to high rates of non-response among such patients. In previous studies, PR prolongation was identified as a strong predictor for CRT response in non-LBBB patients. Interestingly, in our study PR prolongation did not predict the occurrence of VTA, or VTA and death. However the mechanism in which CRT reduces heart failure hospitalization is entirely distinctive than the mechanism in which CRT may be proarrhythmic. In our study, with the utilization of simple RF, we were able to identify a large group of patients without RF, in whom CRT is potentially beneficial and may be associated with an antiarrhythmogenic effect. This group of non-LBBB patients may therefore derive benefit from treatment with cardiac resynchronization therapy. In contrast, in non-LBBB patients with [?] 1 RF, implantation of CRT-D was associated with increased risk of VTA, VTA or death, and appropriate defibrillator shocks, suggesting a possible proarrhytmic effect. Thus, this group of non-LBBB patients may experience improved outcomes with ICD therapy alone rather than combined with cardiac resynchronization therapy.

\section{Limitations}

It should be noted that our findings, based on a subanalysis of the MADIT-CRT trial, should be considered as hypothesis generating since other unmeasured variables in the MADIT CRT data set may have further contributed to the observed outcomes. Accordingly, the present findings need to be further validated in prospective studies that will be appropriately design to identify LBBB patients who may derive benefit vs. harm from CRT implantation. At present, we believe that our data can be used to enhance decision support in this high-risk and understudied population. Furthermore, the current analysis utilizes patient data from MADIT-CRT cohort, with specific enrollment selection criteria. Thus, the findings of the present study are applicable only to patients with mild heart failure symptoms enrolled in the trial.

\section{Conclusions and clinical implications}

Our study suggests that baseline factors that are associated with increased risk for VTA can be used to identify non-LBBB patients in whom CRT-D may associated with beneficial vs. proarrhythmic effects. These findings, if appropriately validated in similar populations of CRT-D recipients and larger studies, may be used for improved selection of patients with non-LBBB for cardiac resynchronization therapy with a defibrillator vs. ICD-only therapy.

\section{Funding source}

The MADIT-CRT study was supported by a research grant from Boston Scientific to the University of Rochester with funds distributed to the coordination and data center, enrolling centers, core laboratories, committees and boards under subcontracts from the University of Rochester.

\section{References}

1. Anand IS, Carson P, Galle E, et al. Cardiac resynchronization therapy reduces the risk of hospitalizations in patients with advanced heart failure: results from the Comparison of Medical Therapy, Pacing and Defibrillation in Heart Failure (COMPANION) trial. Circulation.2009;119(7):969-977.

2. Bradley DJ, Bradley EA, Baughman KL, et al. Cardiac resynchronization and death from progressive heart failure: a meta-analysis of randomized controlled trials. JAMA. 2003;289(6):730-740.

3. Cleland JG, Freemantle N, Erdmann E, et al. Long-term mortality with cardiac resynchronization therapy in the Cardiac Resynchronization-Heart Failure (CARE-HF) trial. Eur J Heart Fail. 2012;14(6):628-634.

4. Brignole M, Auricchio A, Baron-Esquivias G, et al. 2013 ESC Guidelines on cardiac pacing and cardiac resynchronization therapy: the Task Force on cardiac pacing and resynchronization therapy of the European Society of Cardiology (ESC). Developed in collaboration with the European Heart Rhythm Association (EHRA). Eur Heart J.2013;34(29):2281-2329.

5. Bertoldi EG, Polanczyk CA, Cunha V, Ziegelmann PK, Beck-da-Silva L, Rohde LE. Mortality reduction of cardiac resynchronization and implantable cardioverter-defibrillator therapy in heart failure: an updated 
meta-analysis. Does recent evidence change the standard of care? J Card Fail. 2011;17(10):860-866.

6. Kutyifa V, Stockburger M, Daubert JP, et al. PR interval identifies clinical response in patients with non-left bundle branch block: a Multicenter Automatic Defibrillator Implantation Trial-Cardiac Resynchronization Therapy substudy. Circ Arrhythm Electrophysiol.2014;7(4):645-651.

7. Pastore G, Morani G, Maines M, et al. Patients with right bundle branch block and concomitant delayed left ventricular activation respond to cardiac resynchronization therapy. Europace.2018;20(11):e171-e178.

8. Goldenberg I, Kutyifa V, Klein HU, et al. Survival with cardiac-resynchronization therapy in mild heart failure. N Engl J Med. 2014;370(18):1694-1701.

9. Roque C, Trevisi N, Silberbauer J, et al. Electrical storm induced by cardiac resynchronization therapy is determined by pacing on epicardial scar and can be successfully managed by catheter ablation. Circ Arrhythm Electrophysiol. 2014;7(6):1064-1069.

10. Fish JM, Brugada J, Antzelevitch C. Potential proarrhythmic effects of biventricular pacing. J Am Coll Cardiol.2005;46(12):2340-2347.

11. Fish JM, Di Diego JM, Nesterenko V, Antzelevitch C. Epicardial activation of left ventricular wall prolongs QT interval and transmural dispersion of repolarization: implications for biventricular pacing. Circulation. 2004;109(17):2136-2142.

12. Medina-Ravell VA, Lankipalli RS, Yan GX, et al. Effect of epicardial or biventricular pacing to prolong QT interval and increase transmural dispersion of repolarization: does resynchronization therapy pose a risk for patients predisposed to long QT or torsade de pointes? Circulation. 2003;107(5):740-746.

13. Deif B, Ballantyne B, Almehmadi F, et al. Cardiac resynchronization is pro-arrhythmic in the absence of reverse ventricular remodelling: a systematic review and meta-analysis. Cardiovascular research.2018;114(11):1435-1444.

14. Moss AJ, Brown MW, Cannom DS, et al. Multicenter automatic defibrillator implantation trial-cardiac resynchronization therapy (MADIT-CRT): design and clinical protocol. Ann Noninvasive Electrocardiol. 2005;10(4 Suppl):34-43.

15. Cunnington C, Kwok CS, Satchithananda DK, et al. Cardiac resynchronisation therapy is not associated with a reduction in mortality or heart failure hospitalisation in patients with non-left bundle branch block QRS morphology: meta-analysis of randomised controlled trials. Heart. 2015;101(18):1456-1462.

16. Bilchick KC, Kamath S, DiMarco JP, Stukenborg GJ. Bundle-branch block morphology and other predictors of outcome after cardiac resynchronization therapy in Medicare patients. Circulation.2010;122(20):20222030 .

17. Ouellet G, Huang DT, Moss AJ, et al. Effect of cardiac resynchronization therapy on the risk of first and recurrent ventricular tachyarrhythmic events in MADIT-CRT. J Am Coll Cardiol.2012;60(18):1809-1816.

18. Sandhu A, Bao H, Minges KE, et al. Use of Cardiac Resynchronization Therapy Defibrillator in US Hospitals. JAMA Cardiology.2019;4(8):804-809.

19. Jimenez-Candil J, Hernandez J, Perdiguero P, et al. Prognostic Significance of Nonsustained Ventricular Tachycardia Episodes Occurring Early After Implantable Cardioverter-Defibrillator Implantation Among Patients With Left Ventricular Dysfunction. Am J Cardiol.2016;118(10):1503-1510.

20. Singh JP, Hall WJ, McNitt S, et al. Factors influencing appropriate firing of the implanted defibrillator for ventricular tachycardia/fibrillation: findings from the Multicenter Automatic Defibrillator Implantation Trial II (MADIT-II). J Am Coll Cardiol. 2005;46(9):1712-1720.

21. Loring Z, Canos DA, Selzman K, et al. Left Bundle Branch Block Predicts Better Survival in Women Than Men Receiving Cardiac Resynchronization Therapy: Long-Term Follow-Up of 145,000 Patients. 
JACC: Heart Failure. 2013;1(3):237-244.

22. Cheng YJ, Zhang J, Li WJ, et al. More favorable response to cardiac resynchronization therapy in women than in men. Circ Arrhythm Electrophysiol. 2014;7(5):807-815.

\section{Figure Legends}

Figure 1 -

- Figure 1A. Cumulative probability of the primary endpoint (VTA) among non-LBBB patients with CRT-D stratified by the presence or absence of risk factors.

- Figure 1B. Cumulative probability of VTA or death stratified by the presence or absence of risk factors.

- Figure 1C. Cumulative probability of defibrillator appropriate shock therapy stratified by the presence or absence of risk factors.

Figure 2 -

Cumulative probability of; the primary endpoint ventricular tachyarrhythmia (VTA) (left panel); VTA or death (middle panel); and appropriate shock (right panel) among non-LBBB patients with ICD and CRT-D patients stratified by their risk.

Online supplementary Figure A -

Cumulative probability of VTA among non-LBBB patients with ICD, grouped by their Risk Score

Table 1. Multivariate Models* assessing the Risk of different End-points, by the VTA risk factors among CRT-D Patients with non-LBBB

\begin{tabular}{l}
\hline End point \\
\hline Primary End Point: VTA? ¿? \\
One Risk Factors vs. No Risk Factors? ¿? \\
Two Risk Factors vs. No Risk Factors \\
Secondary End Point: Death/ or VTA? ¿? \\
One Risk Factors vs. No Risk Factors? ¿? \\
Two Risk Factors vs. No Risk Factors \\
Secondary End Point: Appropriate Shock Therapy? ¿? \\
One Risk Factors vs. No Risk Factors? ¿? \\
Two Risk Factors vs. No Risk Factors \\
\hline
\end{tabular}

* Models were adjusted for age, gender, QRS length, creatinine, left ventricular ejection fraction, New York Heart Association score, diabetes and ischemic origin.

$\mathrm{CRT}-\mathrm{D}=$ cardiac resynchronization therapy with defibrillator; $\mathrm{ICD}=$ implantable cardioverter defibrillator; $\mathrm{HF}=$ heart failure; $\mathrm{LBBB}=$ left bundle branch block; VTA = ventricular tachyarrhythmia.

Table 2. Multivariate Models* assessing the Effect of CRT-D Versus ICD on different Endpoints, among Patients with non-LBBB, Grouped by their Risk Factors.

\begin{tabular}{lllr}
\hline & & CRT-D with [?] 1 Risk Factor (n. 101) and ICD patients (n. 197) & CRT \\
\hline $\begin{array}{l}\text { End Point } \\
\text { VTA }\end{array}$ & End Point & HR & $95 \%$ \\
Death or VTA & CRT-D vs. ICD & 1.73 & $1.07-$ \\
Appropriate Shock Therapy & CRT-D vs. ICD & 1.72 &
\end{tabular}


* Models were adjusted for age, gender, QRS length, creatinine, blood urea nitrogen, left ventricular ejection fraction, New York Heart Association score, diabetes and ischemic origin.

$\mathrm{CRT}-\mathrm{D}=$ cardiac resynchronization therapy with defibrillator; $\mathrm{ICD}=$ implantable cardioverter defibrillator; $\mathrm{HF}=$ heart failure; $\mathrm{VTA}=$ ventricular tachyarrhythmia.

Figure 1A. Cumulative probability of the primary endpoint (VTA) among non-LBBB patients with CRT-D stratified by the presence or absence of risk factors stratified by their risk.

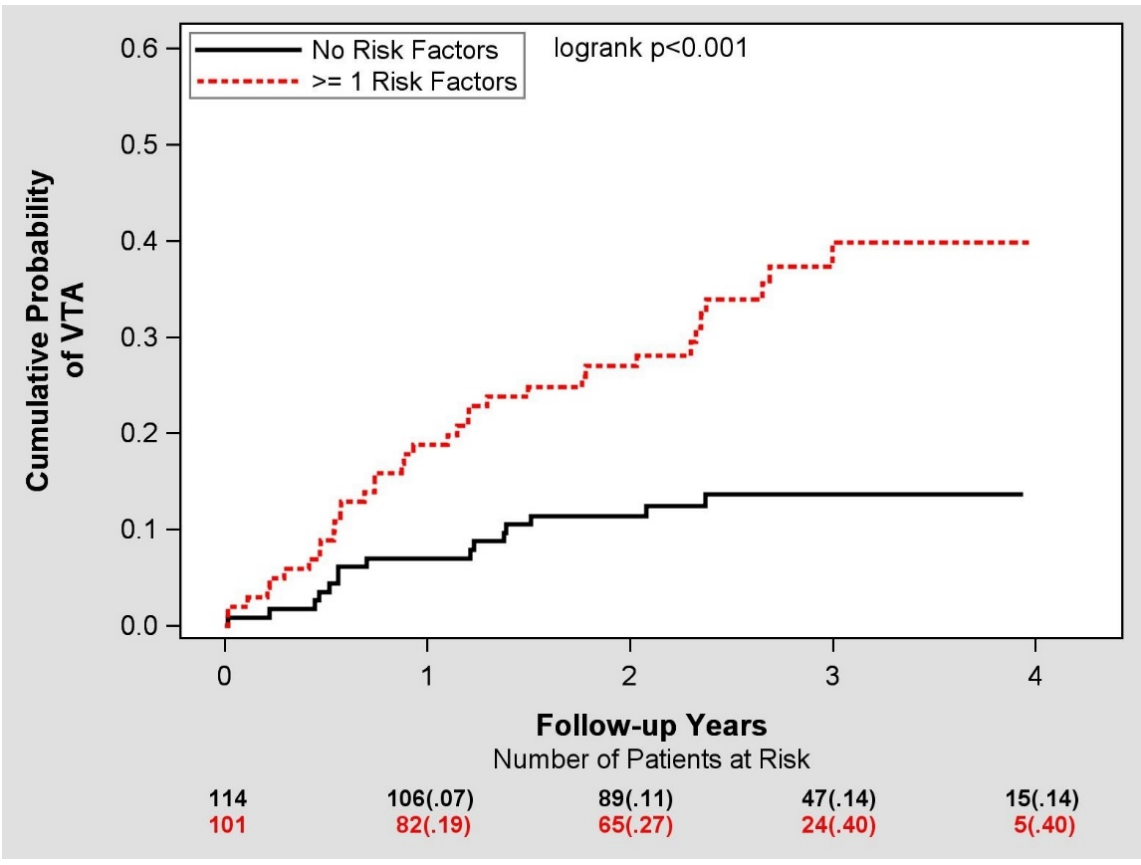

Figure 1B. Cumulative probability of VTA or death stratified by the presence or absence of risk factors. 


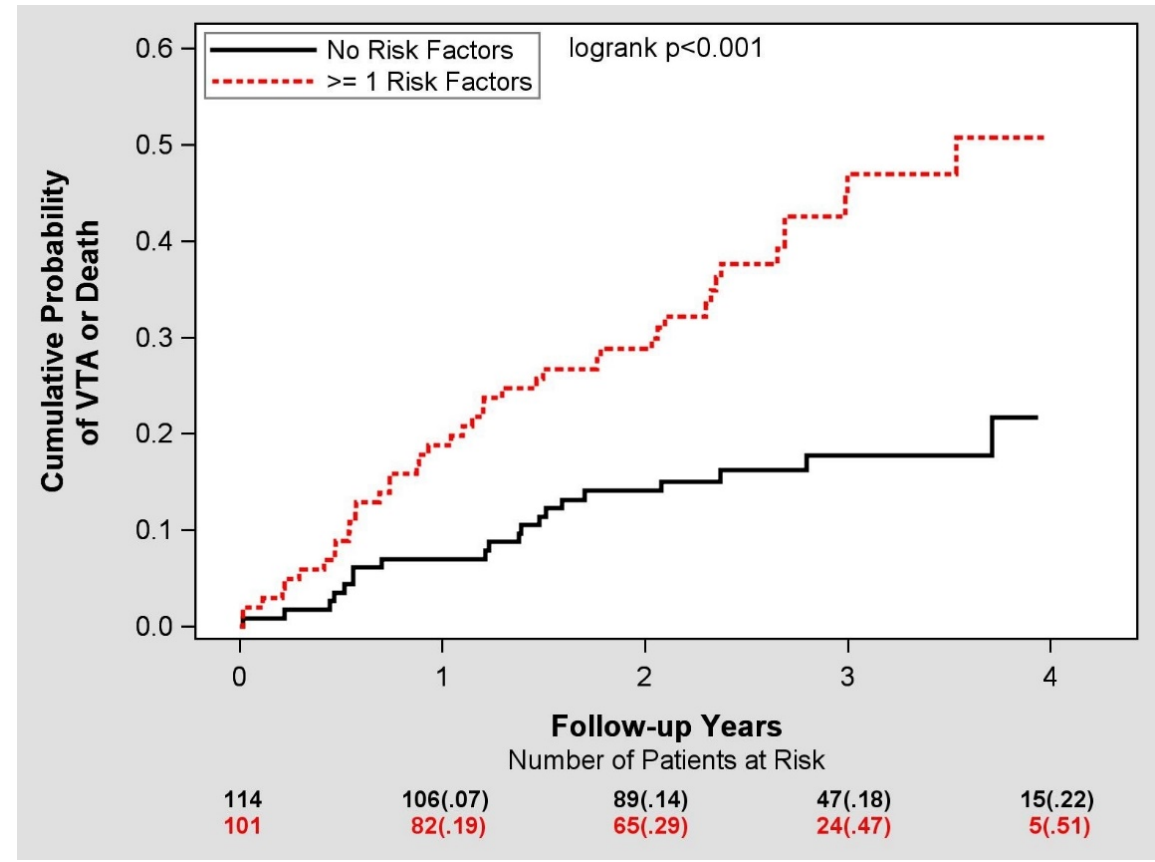

Figure 1C. Cumulative probability of defibrillator appropriate shock therapy stratified by the presence or absence of risk factors.

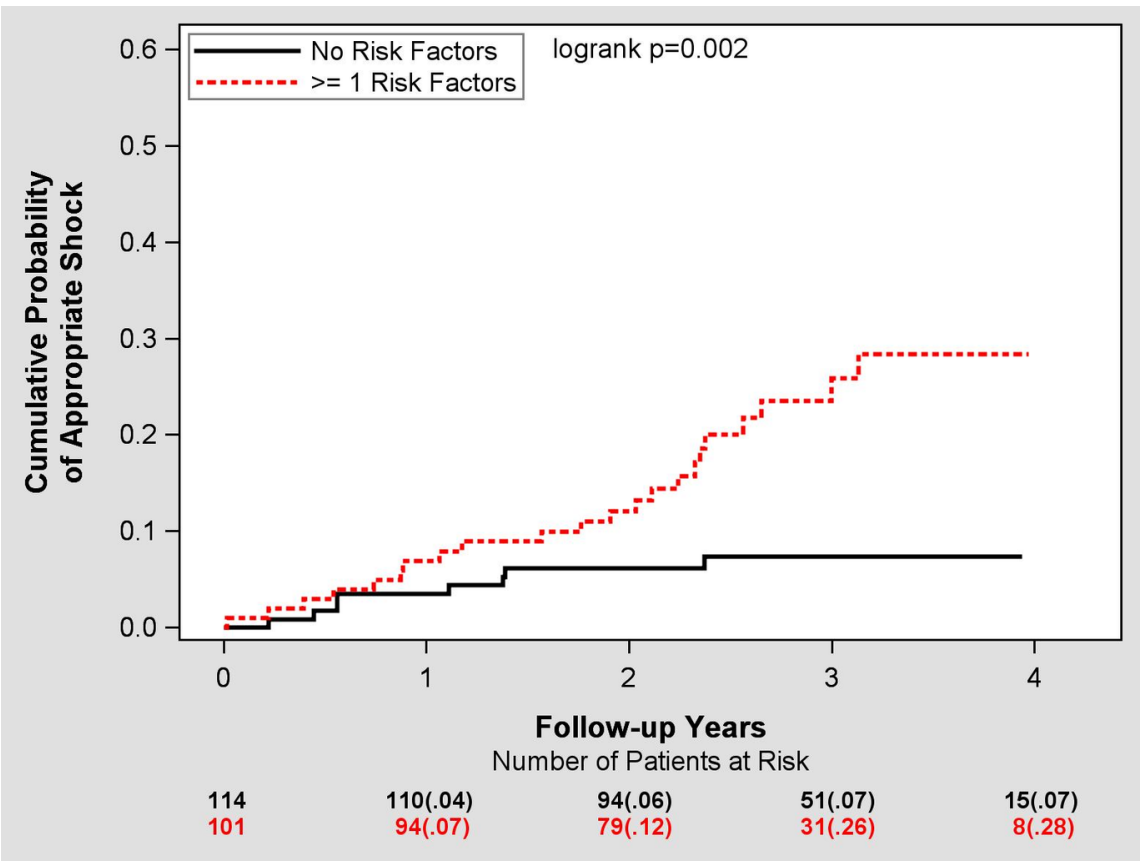

Figure 2 -

Cumulative probability of; the primary endpoint ventricular tachyarrhythmia (VTA) (left panel); VTA or death (middle panel); and appropriate shock (right panel) among non-LBBB patients with ICD and CRT-D patients 

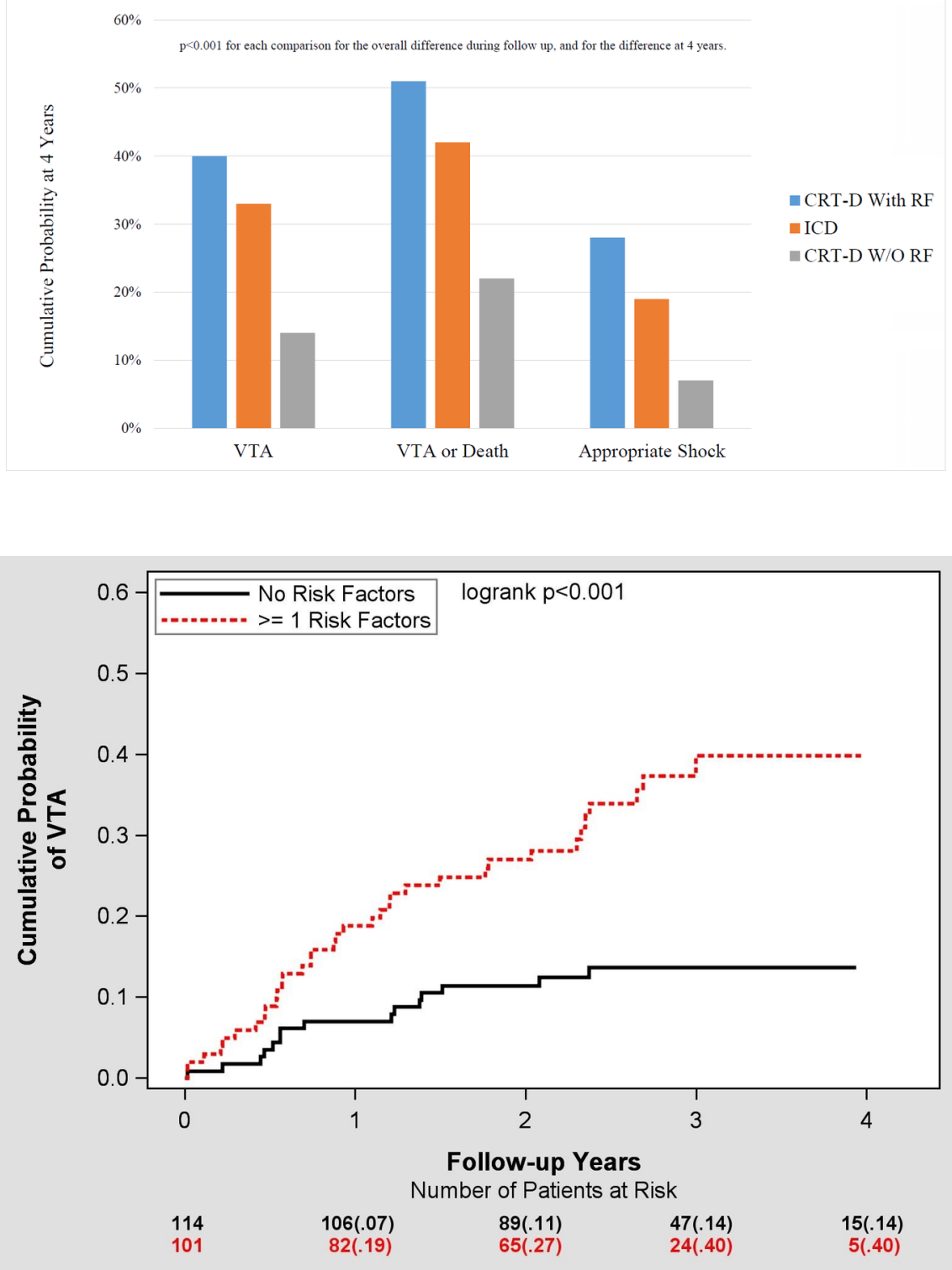

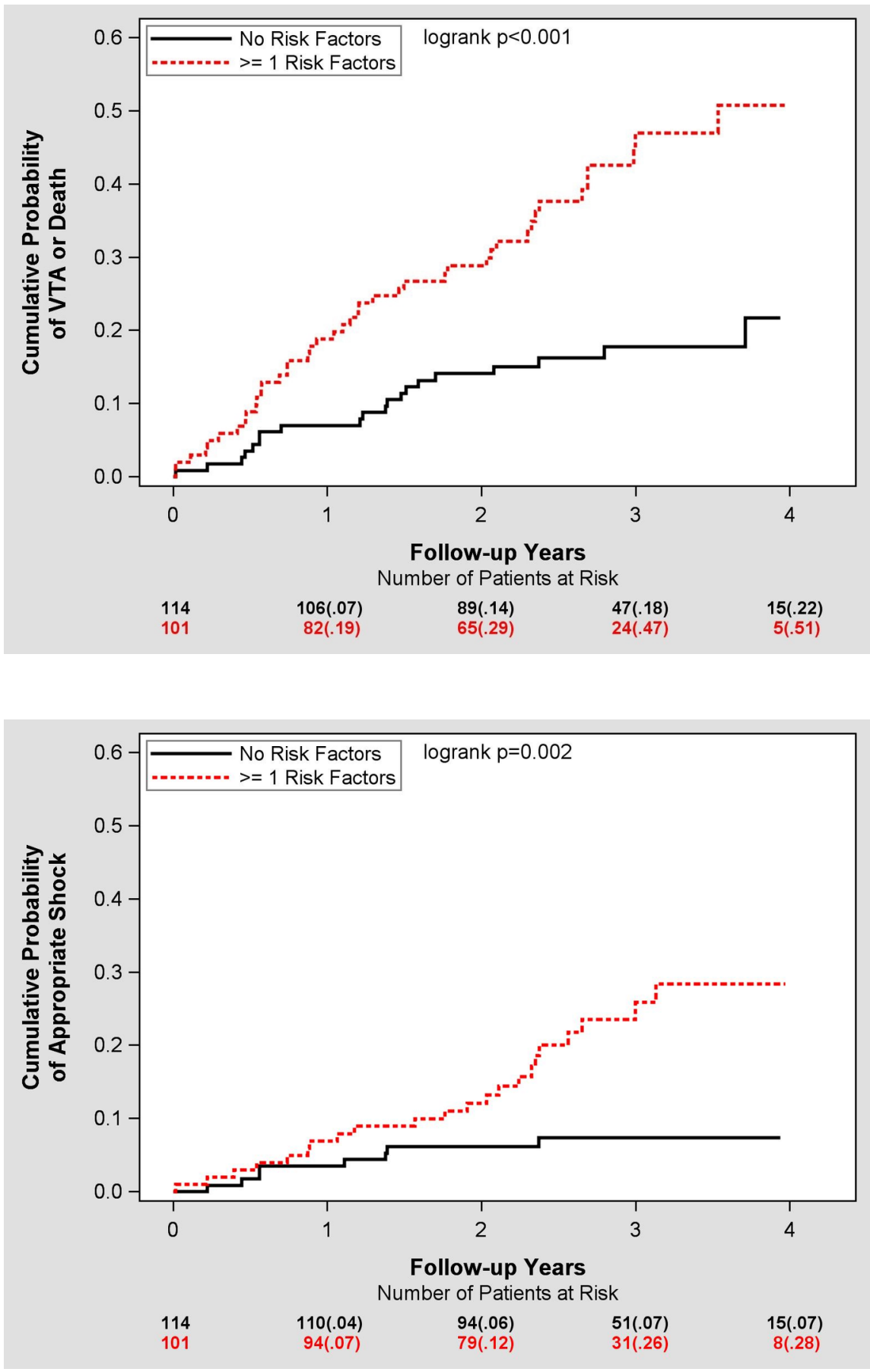


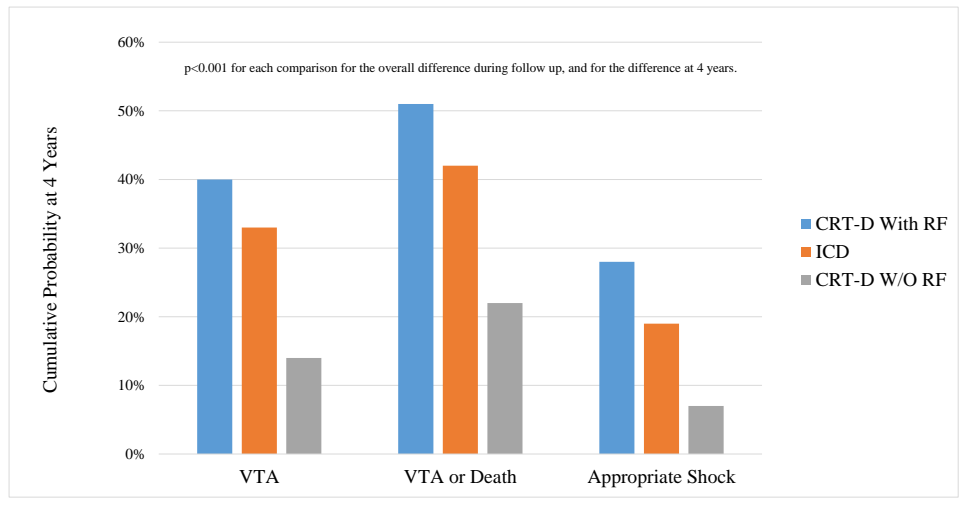

